\title{
Myomectomy scar ectopic pregnancy: diagnostic challenges and management conundrum: a case report
}

\author{
Dhanabagyam Kandaswami ${ }^{1 *}$, Kavitha Jayapal' ${ }^{2}$, Pradeepa Sudhakar ${ }^{3}$, Shyla Usman ${ }^{4}$
}

\author{
${ }^{1}$ Department of Reproductive Medicine, ${ }^{2}$ Department of Obstetrics and Gynecology, Sudha Hospital, Coimbatore, \\ Tamil Nadu, India \\ ${ }^{3}$ Department of Obstetrics and Gynecology, Sudha Hospital, Erode, Tamil Nadu, India \\ ${ }^{4}$ Department of Obstetrics and Gynecology, Sudha Hospital, Chennai, Tamil Nadu, India
}

Received: 06 January 2022

Accepted: 01 February 2022

\section{*Correspondence:}

Dr. Dhanabagyam Kandaswami,

E-mail: sudharesearch2021@gmail.com

Copyright: ( $)$ the author(s), publisher and licensee Medip Academy. This is an open-access article distributed under the terms of the Creative Commons Attribution Non-Commercial License, which permits unrestricted non-commercial use, distribution, and reproduction in any medium, provided the original work is properly cited.

\begin{abstract}
Two patients with a history of previous myomectomy came to the centre for infertility evaluation. Both underwent controlled ovarian stimulation followed by ICSI at our centre. Frozen embryo transfer was done with hormone replacement therapy protocol where two good quality blastocysts were transferred on day 5. After embryo transfer, urine pregnancy tests were positive. But early scans showed pregnancies implanted in the previous scar sites. Laparoscopic removal of the gestational sac was done under ultrasound guidance. This rare form of ectopic pregnancy could occur when the embryo implantation occurs under the serosa at the site of suture failure from the previous surgery. Laparascopic enucleation is the best form of treatment in such cases.
\end{abstract}

Keywords: Myomectomy, Scar ectopic pregnancy, Reproductive, Embryo transfer

\section{INTRODUCTION}

With the advancements in ART, successful live births have been reported in plenty. But one of the most common and sometimes unexpected complications is an ectopic pregnancy. A surgical-site pregnancy is a rare type of ectopic pregnancy, where the pregnancy can occur at the site of a previous surgical scar site. Myomectomy scar pregnancy is characterized by the implantation of the embryo into the site of a previous myomectomy scar. Management of such cases can be done by laparoscopic surgical removal of the gestational sac. Here we report 2 such cases of ectopic pregnancies that resulted from frozen embryo transfer (ET) after myomectomy.

\section{CASE REPORT}

45 years old woman with primary infertility came to our centre. Her initial scans showed multiple myomas. She was posted for Laparoscopic myomectomy. Patient was counselled for donor oocytes programme since her AMH was 0.3 . Frozen embryo transfer was done with hormone replacement therapy protocol after 3 months from myomectomy. 14 days after Embyo Transfer first BetaHCG value was 175. Beta-HCG repeated in 48 hours was 360. A transvaginal ultrasound showed gestational sac in the myomectomy scar region measuring about $3 \times 3 \mathrm{~cm}$. After discussing with senior consultants and experts, patient was initially planned for conservative management. Injection methotrexate was given. There was no expected $15 \%$ reduction in the value of betaHCG after the third dose. 
A transvaginal scan of the patient after the third dose also revealed that size of the sac did not decrease. Hence patient was taken up for laparascopy. $3 \times 4 \mathrm{~cm}$ bulge was seen on the posterior surface of uterus. A $3 \mathrm{~cm}$ incision was made on the uterus just above the intramural pregnancy. Gestational products completely removed and dead space obliteration was done through interrupted sutures in layers. Intra operative blood loss was less than $100 \mathrm{ml}$. Postoperative recovery was uneventful. BetaHCG done on post-operative day 1 was 400 IU/l. Histopathological diagnosis of the specimen showed the presence of villi and decidua. The patient was discharged form hospital after 7 days and followed up in the outpatient department. Beta-HCG decreased to normal 4 weeks after the procedure.
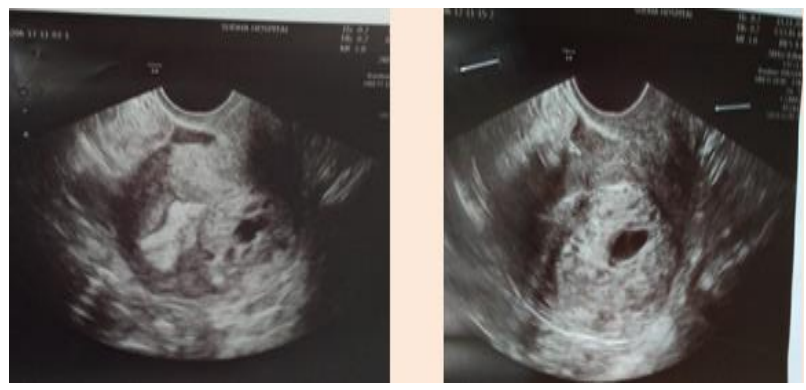

Figure 1: Ultrasonography showing the ectopic gestational sac.

In second case Mrs. Y 33 years, with a history of previous IVF failures came to our centre for secondary infertility evaluation and management. Patient had a history of open myomectomy for a fibroid measuring $4 \mathrm{x} 4$ $\mathrm{cm}$ in 2015. On ultrasound examination during the first visit, there were multiple small intramural fibroids. Laparoscopic myomectomy was proceeded along with adhesiolysis. Patient was counselled for donor oocytes programme considering multiple implantation failures. Frozen embryo transfer was done where 2 high quality blastocysts were tramsferred. Patient tested positive in urine pregnancy test after 14 days. Early scan revealed an intramural pregnancy in the fundus. Patient was taken up for emergency laparoscopy. Intra op sac was seen near the fundus embedded in the interstitium with thinning of serosa. Laparoscopic enucleation was done. Post op uneventful.

\section{DISCUSSION}

Scar ectopic pregnancy is a rare form of abnormal implantation of embryo within the myometrium and the fibrous tissue of the previous scar following uterine surgery like caesarean section, hysterotomy, dilation and curettage, abnormal placentation, myomectomy, metroplasty, and manual removal of placenta. ${ }^{1}$ According to a case report by Tatsuya ishiguro et al this rare form of ectopic pregnancy could have resulted from embryo implantation under the serosa through a micro-sinus tract that was a site of suture failure of the myomectomy scar and was partially affected by the embryo transfer. ${ }^{2}$ Hence it was suggested that after any uterine surgery, it is necessary to evaluate the whole uterus by doing additional imaging, including MRI and a hysteroscopy before embryo transfer to rule out the presence of such sinus tracts. According to a review conducted by Paul et al 68 cases of intramural scar pregnancy were reported. ${ }^{3}$ However, only 3 cases of intramural scar pregnancies after myomectomy were identified. There is a hypothesis that the embryo implants into the myometrium through a microscopic fistula, created through previous uterine surgery, like myomectomy or a caesarean section. ${ }^{4}$ Implantation of the embryo during embryo transfer procedure may also result in development of an intramural pregnancy. The myometrial defect caused from these procedures can cause trophoblast invasion into the myometrium, which in turn can lead to an intramural implantation. ${ }^{5}$

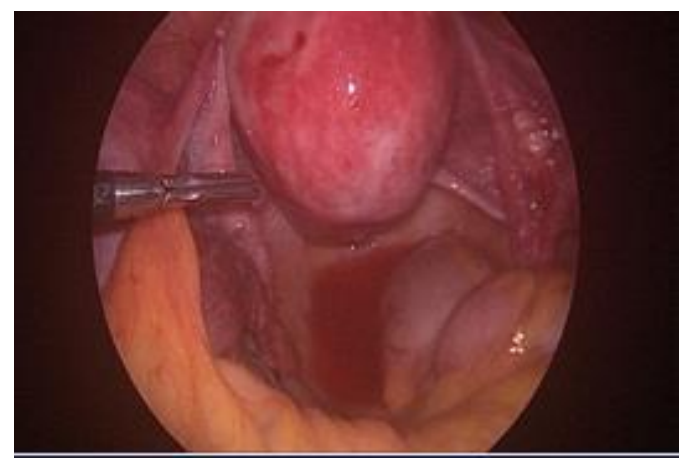

Figure 2: Laproscopic picture showing posterior wall myomectomy scar ectopic pracnancy.

Transvaginal ultrasound is considered the first-line imaging technique for diagnosis of ectopic pregnancy, with a diagnostic accuracy of $90.9 \% .^{6}$ Contrast-enhanced ultrasound may show blood perfusion and identify the implantation site of the embryo. ${ }^{7}$ If diagnosis is made at an early gestation, prior to rupture, conservative options can be used. ${ }^{8}$ There are only limited evidences about managing an intramural ectopic pregnancy. Medical management can be done by injecting localized methotrexate or using systemic methotrexate. Surgical management involves removal of the products of conception. Treatment is dependent on location, extent of myometrial involvement, gestational age at diagnosis and desires for future fertility. ${ }^{1}$ Medical management with local and systemic multidose methotrexate cannot be done in advanced gestational age. Wedge resection of the uterus cannot be done if the location and size of the intramural ectopic pregnancy would require a large amount of uterine tissue to be excised. ${ }^{1}$ In a recent study it was shown that the use of systemic methotrexate or intra-sac methotrexate (with potassium chloride if embryonic cardiac activity is present) can be done for such ectopic pregnancies, especially in those who desire to preserve fertility. ${ }^{9}$

Surgical management of benign asymptomatic fibroids is controversial, with the general consensus being against 
surgery if patients are asymptomatic. Hence in these patients the previous surgeries for fibroids could have been avoided if it had not caused any adverse effects. ${ }^{10}$ Early diagnosis is the only way to prevent complications like uterine rupture. Failure to diagnose an intramural pregnancy can result in severe bleeding due to the proximity of the gestational sac to the intramyometrial vasculature. ${ }^{11}$ Tight sutures of the uterine wall incision, including the endometrium, myometrium and serosa during myomectomy, could possibly reduce the incidence of such myometrial scar ectopic pregnancies. ${ }^{12}$ The paper by Leyder et al reports a pregnancy within a previously necrotized fibroid. She had a history of uterine artery embolisation for the fibroid and then underwent frozen embryo transfer. So the risk of an ectopic pregnancy within the myometrium has to be considered even after uterine artery embolisation. ${ }^{13}$ Final management option should be individualised according to patient factors, with multidisciplinary approach with TVS ultrasound playing a pivotal role. But due to the limited number of cases reported, specific mode of treatment recommendations to prevent it are not currently available. More detailed case reports are needed to make such guidelines.

\section{CONCLUSION}

Clinicians should always consider the possibility of an ectopic implantation of the sac after any kind of uterine surgery. Although myomectomy scar ectopic pregnancy is rare, early scans and diagnosis can prevent catastrophic obstetric emergencies.

\section{Funding: No funding sources \\ Conflict of interest: None declared \\ Ethical approval: Not required}

\section{REFERENCES}

1. Bannon K, Fernandez C, Rojas D, Levine EM, Locher S. Diagnosis and management of intramural ectopic pregnancy. J Minim Invasive Gynecol. 2013; 20(5):697-700.

2. Ishiguro T, Yamawaki K, Chihara M, Nishikawa N, Enomoto T. Myomectomy scar ectopic pregnancy following a cryopreserved embryo transfer. Reprod Med Biol. 2018;17(4):509-13.

3. Zhu L, Yang X, Sun W, Qian L, Li S, Li D. Myomectomy scar pregnancy: a case report and review of the literature. J Int Med Res. 2020;48(7): $30-7$.
4. Karakök M, Balat O, Sari I, Kocer NE, Erdogan R. Early diagnosed intramural ectopic pregnancy associated with adenomyosis: report of an unusual case. Clin Exp Obstet Gynecol. 2002;29:217-8.

5. Khalifa Y, Redgment CJ, Yazdani N, Taranissi M, Craft IL. Intramural pregnancy following difficult embryo transfer. Hum Reprod. 1994;9:2427-8.

6. Abdel-Gadir A, Shah K, Oyawoye OO, Chander BP. Subserosal intramural ectopic pregnancy in an adenomyotic area following assisted reproduction treatment. Gynecol Surg. 2009;6:267-71

7. Xiong X, Yan P, Gao C. The value of contrastenhanced ultrasound in the diagnosis of cesarean scar pregnancy. Biomed Res Int. 2016;2016:476-85.

8. Kirk E, McDonald K, Ress J, Govind A. Intramural ectopic pregnancy: a case and review of the literature. Eur J Obstet Gynaecol Reprod Biol. 2013; 168:129-33.

9. Ramkrishna J, Kan GR, Reidy KL, Ang WC, PalmaDias R. Comparison of management regimens following ultrasound diagnosis of nontubal ectopic pregnancies: a retrospective cohort study. BJOG. 2018;125:567-75.

10. Abdel-Gadir A, Shah K, Oyawoye OO, Chander BP. Subserosal intramural ectopic pregnancy in an adenomyotic area following assisted reproduction treatment. Gynecol Surg. 2009;6:267-71.

11. Su S, Chavan D, Song K. Distinguishing between intramural pregnancy and choriocarcinoma: a case report. Oncol Lett. 2017;13:2129-32.

12. Zhu L, Yang X, Sun W, Qian L, Li S, Li D. Myomectomy scar pregnancy: a case report and review of the literature. J Int Med Res. 2020; 48(7):300-9.

13. Leyder M, De Vos M, Popovic-Todorovic B, Dujardin M, Devroey P, Fatemi HM. Intramyometrial ectopic pregnancy in an ICSI patient following uterine artery embolization. Reprod Biomed Online. 2010;20(6):831-5.

Cite this article as: Kandaswami D, Jayapal K, Sudhakar P, Usman S. Myomectomy scar ectopic pregnancy: diagnostic challenges and management conundrum: a case report. Int J Reprod Contracept Obstet Gynecol 2022;11:954-6. 